EESTI NSV TEADUSTE AKADEEMIA TOIMETISED, 31. KOIDE

FOOSIKA * MATEMAATIKA. 1982, NR. 1

ИЗВЕСТИЯ АКАДЕМИИ НАУК ЭСТОНСКОП ССР. ТОМ 31 ФИЗИКА * МАТЕМАТИКА. 1982, № 1

\title{
COMPARISON THEOREMS OF SUMMATION METHODS WITH ESTIMATIONS OF CONSTANTS
}

(Presented by A. Humal)

1. Introduction. This paper deals with the approximation by summation methods of Fourier expansions in Banach spaces. It is supposed that in this Banach space is given a sequence of projections, which satisfies inequalities of Bernstein-type and multiplier type. We shall give a comparison theorem between the approximation orders of a pair of summation methods. Certain novelty is that the constants in this comparison theorem have an explicit form.

Let $\mathbf{P}, \mathbf{N}$ be the sets of all non-negative and of all positive integers, respectively. Let $X$ be an arbitrary (real or complex) Banach space with norm $\|$ o $\|$ and let $[X]$ be the Banach algebra of all bounded linear operators of $X$ into itself. Let $\left\{P_{k}: k \in \mathbf{P}\right\}$ be a total sequence of mutually orthogonal continuous projections on $X$, i. e.,

i) $P_{k} \in[X]$ for each $k \in \mathbf{P}$,

ii) if $P_{k} f=0$ for all $k \in \mathbf{P}$, then $f=0$,

iii) $P_{j} P_{k}=\delta_{j k} P_{k}$, where $\delta_{j k}$ is Kronecker's symbol.

Suppose that the space $X$ and the sequence $\left\{P_{k}\right\}$ are such that the following conditions are satisfied.

For some non-negative function $\varphi$ on $\mathbf{P}$ let there exist constants $M_{1}$, $M_{2}>0$, such that for every $f \in X$ and $v, n \in \mathbf{N}$ the inequalities

$$
\begin{aligned}
& \left\|\sum_{k=0}^{n} \varphi(k)^{v} P_{k} f\right\| \leqslant M_{1} \varphi(n)^{v}\left\|\sum_{k=0}^{n} P_{k} f\right\|, \\
& \left\|\sum_{k=0}^{n} \varphi(k)^{-v} P_{k} f\right\| \leqslant M_{2}\left\|\sum_{k=0}^{n} P_{k} f\right\|,
\end{aligned}
$$

are satisfied.

Note that the inequality (B) is an inequality of Bernstein-type and the inequality $(M)$ is an inequality of multiplier-type.

Example 1. Let $X=X_{2 \pi}:=L_{2 \pi}^{p_{1}}, 1 \leqslant p<\infty$, or : $=C_{2 \pi}$ be the Banach space of $2 \pi$-periodic functions with standard norms $\|\cdot\|_{X_{2 \pi}}$. Define $\left\{P_{k}\right\}$ by

$$
P_{0} f(x):=\hat{f}(0), \quad P_{k} f(x):=\hat{f}(k) e^{i k x}+\hat{f}(-k) e^{-i k x} \quad(k \in \mathbf{N}),
$$

where $\hat{f}(k)$ are the usual Fourier coefficients of $f \in X_{2 \pi}$. Then for $\varphi(k)=$ $=|k|$ the inequality (B) is satisfied as follows (cf. $\left[{ }^{1}\right], \mathrm{n}^{\mathrm{p}} 84$ ):

$$
\left\|\sum_{k=-n}^{n}|k| v \hat{f}(k) e^{i k}\right\|_{X_{2 \pi}} \leqslant n^{v}\left\|\sum_{k=-n}^{n} \hat{f}(k) e^{i k \circ}\right\|_{X_{2 \pi}} .
$$


Ex a m ple 2. Let us assume that the operator $S_{n}$ defined as

$$
S_{n} f:=\sum_{k=0}^{n} P_{k} f \quad(f \in X)
$$

is uniformly bounded, i. e.,

$$
\left\|S_{n} f\right\| \leqslant B\|f\|,
$$

where the constant $B$ is independent of $n \in \mathbf{P}$ and $f \in X$. Then by the results of $\left[{ }^{2}\right]$ (applying the inequality $(3.1)$ and the theorems $2.1,2.2$ ), we have for every $\lambda>0, f \in X$

$$
\left\|\sum_{k=0}^{n} k^{\lambda} P_{k} f\right\| \leqslant 2 B^{2} n^{\lambda}\left\|\sum_{k=0}^{n} P_{k} f\right\| .
$$

Example 3. Let us introduce the $n$-th Cesàro mean operator (of order 1)

Assume that

$$
(C, 1)_{n} f:=\sum_{k=0}^{n}\left(1-\frac{k}{n+1}\right) P_{k} f \quad(f \in X) .
$$

$$
\left\|(C, 1)_{n} f\right\| \leqslant C\|f\|,
$$

where the constant $C>0$ in independent of $n \in \mathbf{P}$ and $f \in X$. Then according to the results of $\left[{ }^{3}\right]$ (applying the theorem 3.2), we have for every $\lambda>0$ that

$$
\left\|\sum_{h=1}^{n} k^{-\lambda} P_{k} f\right\| \leqslant 4 C\left\|\sum_{k=0}^{n} P_{k} f\right\| .
$$

It is well known that if in addition $X=X_{2 \pi}$ and $\left\{P_{k}\right\}$ are defined by (1.1), then the condition (1.2) is satisfied with $C=1$.

According to these examples both inequalities (B) and (M) are satisfied in the trigonometrical case. In general case, the validity of the inequality (B) is unknown because we demand that the constant $M_{1}$ must be independent of $v \in \mathbf{N}\left[{ }^{2}\right]$.

For each $f \in X$ one may associate its unique Fourier expansion

$$
f \sim \sum_{k=0}^{\infty} P_{k} f
$$

and a multiplier operator $U_{r} \in[X]$ defined by expansion

$$
U_{r} f \sim \sum_{k=0}^{\infty} u_{k}(r) P_{k} f \quad(r \in R) .
$$

Let. $V_{r} \in[X]$ be another multiplier operator generated by another sequence $v(r)=\left\{v_{k}(r): k \in \mathbf{P}\right\}$. The problem is to find an inequality

$$
\left\|f-U_{r} f\right\| \leqslant K\left\|f-V_{r} f\right\|,
$$

where the constant $K>0$ is independent of $f \in X$ and $r \in R$.

Initially this problem was presented by J. Favard [4,5]. Since 1968 by H. S. Shapiro $\left[{ }^{6}\right]$ and R. M. Trigub $\left[{ }^{7}\right]$, followed by P. L. Butzer, R. J. Nessel, W. Trebels $\left[{ }^{3}\right]$, M. Zamansky $\left[{ }^{8}\right]$, etc.; the comparison theorems of two different approximation processes are discussed very extensively and quite thoroughly. We shall derive the inequality (1.3) by a method, which is comparatively simple and practically applicable. This method enables also to estimate the constant $K$. 
2. A comparison theorem. Let us begin with the definition of condition $A$ (analyticity) for multiplier sequences $u(r)=\left\{u_{k}(r)\right\}, v(r)=$ $=\left\{v_{k}(r)\right\}$ having the generated multiplier operators $U_{r}, V_{r} \in[X]$, respectively.

Definition. The pair of multiplier sequences $(u(r), v(r))$ is said to satisfy the condition $A$ if there exists $N \in \mathbf{N}$, a function $\varphi \geqslant 0$ on $\mathbf{P}$ and coefficients $c_{v}$ (real or complex) such that for every $0 \leqslant k \leqslant N$ the expansion

$$
1-u_{k}(r)=\left[1-v_{k}(r)\right] \sum_{v=-\infty}^{\infty} c_{v}(r) \varphi(k)^{v}
$$

holds.

Next we shall introduce the best approximation by elements of subspace $\Pi_{n} \subset X$, where

$$
\Pi_{n}:=\left\{f \in X: f=P_{0} f+P_{1} f+\ldots+P_{n} f\right\} .
$$

As usually, the best approximation of $f \in X$ by elements $t_{n} \in \Pi_{n}$ can be defined by

$$
E_{n}(f):=\inf _{t_{n} \in \Pi_{n}}\left\|f-t_{n}\right\| .
$$

An element $\dot{t}_{n} \in \Pi_{n}$ for which the infimum (2.1) is attained, is called an element of the best approximation to $f \in X$. We shall assume that $\dot{t}_{n}^{*}$ exists for each $f \in X$.

Now we can formulate our main result.

Theorem. Let $X,\left\{P_{k}\right\}$ satisfy inequalities (B) and (M). Let the pair of multiplier sequences $(u(r), v(r))$ satisfy condition $A$ and let $U_{r}, V_{r} \in$ $\in[X](r \in R)$ be two families of multiplier operators which are given by $u(r), v(r)$, respectively. Then for each $f \in X$ and $r \in R$

$$
\begin{gathered}
\left\|f-U_{r} f\right\| \leqslant\left(1+\left\|U_{r}\right\|\right) E_{N}(f)+ \\
+G(u, v ; r, N)\left\{\left(1+\left\|V_{r}\right\|\right) E_{N}(f)+\left\|f-V_{r} f\right\|\right\},
\end{gathered}
$$

where

$$
G(u, v ; r, N):=\left|c_{0}(r)\right|+M_{1} \sum_{v=1}^{\infty}\left|c_{v}(r)\right| \varphi(N)^{v}+M_{2} \sum_{v=1}^{\infty}\left|c_{-v}(r)\right| .
$$

Proof. Let $t_{N} \in \Pi_{N}$ be the element of the best approximation to $f \in X$. Using the triangle inequality, we have

$$
\left\|f-U_{r} f\right\| \leqslant\left(1+\left\|U_{r}\right\|\right) E_{N}(f)+\left\|t_{N}^{*}-U_{r} t_{N}^{*}\right\| .
$$

Due to condition $A$,

$$
\begin{gathered}
\dot{t_{N}}-U_{r} t_{N}^{*}=\sum_{k=0}^{N}\left[1-u_{k}(r)\right] P_{k} t_{N}^{*}=c_{0}(r) \sum_{k=0}^{N}\left[1-v_{k}(r)\right] P_{k} t_{N}^{*}+ \\
+\sum_{v=1}^{\infty} c_{v}(r) \sum_{k=0}^{N} \varphi(k)^{v}\left[1-v_{k}(r)\right] P_{k} t_{N}^{*}+\sum_{v=1}^{\infty} c_{-v}(r) \sum_{k=0}^{N} \varphi(k)^{-v}\left[1-v_{k}(r)\right] P_{k} t_{N}^{*} .
\end{gathered}
$$

In addition, from the inequalities $(B)$ and $(M)$ it follows that

$$
\begin{gathered}
\left\|t_{N}^{*}-U_{r} t_{N}\right\| \leqslant\left|c_{0}(r)\right|\left\|t_{N}^{*}-V_{r} t_{N}^{*}\right\|+\sum_{v=1}^{\infty}\left|c_{v}(r)\right| \varphi(N)^{v} M_{1}\left\|t_{N}^{*}-V_{r} t_{N}^{*}\right\|+ \\
+\sum_{v=1}^{\infty}\left|c_{-v}(r)\right| M_{2}\left\|t_{N}^{*}-V_{r} t_{N}^{*}\right\|=G(u, v ; r, N)\left\|t_{N}^{*}-V_{r} t_{N}^{*}\right\|,
\end{gathered}
$$


where $G(u, v ; r, N)$ is given by (2.2). According to the obvious inequality

$$
\left\|t_{N}^{*}-V_{r} t_{N}^{*}\right\| \leqslant\left(1+\left\|V_{r}\right\|\right) E_{N}(f)+\left\|f-V_{r} t\right\|
$$

we have

$$
\left\|t_{N}^{*}-U_{r} t_{N}^{*}\right\| \leqslant G(u, v ; r, N)\left\{\left(1+\left\|V_{r}\right\|\right) E_{N}(f)+\left\|f-V_{r} f\right\|\right\} .
$$

Therefore, inequality (2.3) proves the Theorem.

Following the proof of the Theorem we may draw some remarks.

i) If in condition $A c_{-v}(r)=0$ for every $v \in \mathbf{N}$, then the Theorem holds without inequality $(M)$.

ii) If in condition $A$ instead of infinite series are finite sums, then the constants $M_{1}, M_{2}$ in inequalities (B), (M), respectively, can depend on $v \in \mathbf{N}$.

If $V_{r}$ are polynomial (i. e. $r:=n \in \mathbf{P}$ and $V_{n} f \in \Pi_{n}$ for each $f \in X$ ), then from the inequality $E_{n}(f) \leqslant\left\|f-V_{n} f\right\|$ we immediately have

Corollary 1. If $V_{n}$ are polynomial, then under the assumptions of the Theorem for each $f \in X$ and $n \in \mathbf{N}$

$$
\left\|f-U_{n} f\right\| \leqslant\left\{1+\left\|U_{n}\right\|+G(u, v ; n, n)\left(2+\left\|V_{n}\right\|\right)\right\}\left\|f-V_{n} f\right\| .
$$

3. Applicati_ons. The simplest multiplier operators are the $Z y g$ mund typical means $Z_{n}^{\lambda}$ of order $\lambda>0$ defined by multiplier sequence

$$
z_{k}^{\lambda}(n):=\left\{\begin{array}{cc}
1-\left(\frac{k}{n+1}\right)^{\lambda}, & 0 \leqslant k \leqslant n, \\
0, & k>n .
\end{array}\right.
$$

It is known that the approximation order by operators $Z_{n}^{\lambda}$ essentially depends on $\lambda$. Also, the operators $Z_{n}^{\lambda}$ can be used to study the order of approximation as scale-means.

We shall give some applications of the comparison theorem if one of the multiplier sequences $u(r)$ (or $v(r)$ ) is the Zygmund multiplier sequence $z^{\lambda}(n):=\left\{z_{k}^{\lambda}(n)\right\}$. If $v(n)=z^{\lambda}(n)$ and $u(r)$ is arbitrary, another framework was given by the author in $\left[{ }^{9}\right]$. In the following examples we shall give only the function $G(u, v ; r, N)$ as defined by $(2.2)$, where $\varphi(N)=N$. The details we shall give only in examples $4,5,10$, the other examples can be handled by the same way. Our proofs are based on the Maclaurin's series of functions $h(k):=\left[1-u_{k}(r)\right] /\left[1-v_{k}(r)\right]$ or $1 / h(k)$. The definitions of summation methods contained in the next examples one can find in $\left[{ }^{10}\right]$.

$\mathrm{Ex}$ a m ple 4. It will be shown that the estimation

$$
G\left(z^{\lambda}, z^{\mu} ; n, n\right) \leqslant\left\{\begin{array}{cc}
M_{1}, & \mu<\lambda, \\
1, & \mu=\lambda, \\
M_{2}(n+1)^{\mu-\lambda}, & \mu>\lambda,
\end{array}\right.
$$

is valid. Indeed, for the multiplier sequences $z^{\lambda}(n), z^{\mu}(n)$ one has

$$
1-z_{k}^{\lambda}(n)=\left[1-z_{k}^{\mu}(n)\right](k /(n+1))^{\lambda-\mu} \quad(0 \leqslant k \leqslant n) .
$$

Then, according to (A), the function $(2.2)$ is bounded. We need the following

$\mathrm{Le} \mathrm{m} \mathrm{m}$ a. If the inequality (1.2) holds, then

$$
\left\|Z_{n}^{\lambda}\right\| \leqslant(2 \lambda-1) C \quad(\lambda \in \mathbf{N}) .
$$


Proof. Apply Abel's transformation twice to $Z_{n}^{\lambda}$ and use (1.2). In the trigonometrical case $M_{1}=1$ (example 1) and $M_{2}=4$ (example 3). Now by Corollary 1 we have

Corollary 2. For each $f \in X_{2 \pi}, \lambda, \mu, n \in \mathbf{N}$

$$
\left\|f-Z_{n}^{\lambda} f\right\|_{X_{2 \pi}} \leqslant\left\|f-Z_{n}^{\mu} f\right\|_{X_{2 \pi}} \times \begin{cases}2(\lambda+\mu)+1, & \mu<\lambda, \\ 2 \lambda+4(n+1)^{\mu-\lambda}(1+2 \mu), & \mu>\lambda .\end{cases}
$$

Example 5. The Bernstein-Rogosinski method $\mathcal{B}_{n}$ is defined by multiplier $b_{k}(n):=\cos (k \pi /(2 n+1))$ for $k \leqslant n$ and $:=0$ for $k>n$. Then for $0 \leqslant k \leqslant n$

$$
1-b_{k}(n)=\left[1-z_{k}^{2}(n)\right] \pi^{2}\left(\frac{n+1}{2 n+1}\right)^{2} \sum_{v=0}^{\infty} \frac{(-1)^{v}}{(2 v+2) !}\left(\frac{k \pi}{2 n+1}\right)^{2 v}
$$

and

$$
\begin{aligned}
G\left(b, z^{2} ; n, n\right)=\frac{\pi^{2}}{2}\left(\frac{n+1}{2 n+1}\right)^{2} & +M_{1}\left(\frac{n+1}{n}\right)^{2}\left(\operatorname{ch} \frac{n \pi}{2 n+1}-\frac{1}{2}\left(\frac{n \pi}{2 n+1}\right)^{2}-1\right) \leqslant \\
& \leqslant \frac{2}{9} \pi^{2}+4 M_{1}\left(\operatorname{ch} \frac{\pi}{2}-\frac{\pi^{2}}{8}-1\right) \approx 2,18+1,16 M_{1} .
\end{aligned}
$$

On the contrary

$$
1-z_{k}^{2}(n)=\left[1-b_{k}(n)\right] \cdot \frac{2}{\pi^{2}}\left(\frac{2 n+1}{n+1}\right)^{2}\left[1+\sum_{v=1}^{\infty} \frac{B_{v}(2 v-1)}{(2 v) !}\left(\frac{k \pi}{2 n+1}\right)^{2 v}\right],
$$

where the coefficients $B_{v}$ are the Bernoulli numbers. Then from (2.2) it follows that

$$
\begin{aligned}
G\left(z^{2}, b ; n, n\right) & =\frac{2}{\pi^{2}}\left(\frac{2 n+1}{n+1}\right)^{2}\left[1-M_{1}+M_{1}\left(\frac{n \pi}{2(2 n+1)}\right)^{2} \sin ^{-2} \frac{n \pi}{2(2 n+1)}\right] \leqslant \\
& \leqslant 8\left(1-M_{1}\right) / \pi^{2}+M_{1} \approx 0,81+0,19 M_{1} .
\end{aligned}
$$

Example 6. The Jackson-de La Vallée Poussin method $V_{n}$ is given by $v_{k}(n):=1-(3 / 2)(k / n)^{2}+(3 / 4)(k / n)^{3}$ for $k \leqslant n,:=(1 / 4)(2-k / n)^{3}$ for $n<k \leqslant 2 n-1$ and $:=0$ for $k>2 n-1$. Then we have

$$
\begin{aligned}
& G\left(v, z^{2} ; n, n\right)=\frac{3}{4}\left(\frac{n+1}{n}\right)^{2}\left(2+M_{1}\right) \leqslant 6+3 M_{1}, \\
& G\left(z^{2}, v ; n, n\right)=\frac{2}{3}\left(\frac{n}{n+1}\right)^{2}\left(1+M_{1}\right) \leqslant \frac{2}{3}\left(1+M_{1}\right) .
\end{aligned}
$$

Example 7. The Jackson method $J_{n}$ is given by

$$
j_{k}(n):=\left\{\begin{array}{lr}
1-\frac{3 k}{2 n\left(2 n^{2}+1\right)}-\frac{3 k^{2}}{2 n^{2}+1}+\frac{3 k^{3}}{2 n\left(2 n^{2}+1\right)}, & 0 \leqslant k \leqslant n, \\
2 n^{2}-1-\frac{k\left(12 n^{2}-1\right)}{2 n\left(2 n^{2}+1\right)}+\frac{3 k^{2}}{2 n^{2}+1}-\frac{k^{3}}{2 n\left(2 n^{2}+1\right)}, & n<k \leqslant 2 n-2, \\
0, & k>2 n-2 .
\end{array}\right.
$$

We obtain that 


$$
G\left(j, z^{2} ; n, n\right)=\frac{3(n+1)^{2}}{2\left(2 n^{2}+1\right)}\left(\frac{M_{2}}{n}+2+M_{1}\right) \leqslant 2\left(2+M_{1}+M_{2}\right) .
$$

To find $G\left(z^{2}, j ; n, n\right)$, let us apply decomposition into partial fractions. Hence

$$
\begin{aligned}
\frac{1-z_{k}^{2}(n)}{1-j_{k}(n)} & =\frac{2\left(2 n^{2}+1\right)}{3(n+1)^{2}} \cdot \frac{k n}{1+2 k n-k^{2}}= \\
& =\frac{2 n^{2}+1}{3(n+1)^{2}} \cdot \frac{n}{\sqrt{n^{2}+1}}\left(\frac{k_{0}}{k_{0}-k}-\frac{1}{k_{0} k+1}\right),
\end{aligned}
$$

where $k_{0}=n+\sqrt{n^{2}+1}$. Since $0 \leqslant k \leqslant n<k_{0}$, it follows that

$$
\begin{aligned}
G\left(z^{2}, j ; n, n\right) & =\frac{2 n^{2}+1}{3(n+1)^{2}} \cdot \frac{n}{\sqrt{n^{2}+1}}\left(1+M_{1} \frac{n}{\sqrt{n^{2}+1}}+M_{2} \frac{1}{n+\sqrt{n^{2}+1}-1}\right) \leqslant \\
& \leqslant\left(2+2 M_{1}+\sqrt{2} M_{2}\right) / 3 .
\end{aligned}
$$

Example 8. The Abel-Cartwright method $A_{n}$ is given by $a_{k}^{\lambda}(n):=$ $=\exp \left(-(k /(n+1))^{\lambda}\right)$ for $\lambda>0$. We can show that

$$
\begin{aligned}
G\left(a^{\lambda}, z^{\lambda} ; n, n\right) & =1+M_{1} \sum_{v=1}^{\infty} \frac{1}{(v+1) !}\left(\frac{n}{n+1}\right)^{\lambda v}= \\
& =1+M_{1}\left(\frac{n+1}{n}\right)^{\lambda}\left(\exp \left(\frac{n}{n+1}\right)^{\lambda}-\left(\frac{n}{n+1}\right)^{\lambda}-1\right) \leqslant \\
& \leqslant 1+M_{1}(e-2), \\
G\left(z^{\lambda}, a^{\lambda} ; n, n\right) & =1+\frac{M_{1}}{2}\left(\frac{n}{n+1}\right)^{\lambda}+\sum_{v=1}^{\infty} \frac{B_{v}}{(2 v) !}\left(\frac{n}{n+1}\right)^{2 \lambda v}= \\
& =1+\frac{M_{1}}{2}\left(\frac{n}{n+1}\right)^{\lambda}+M_{1}\left(1-\frac{1}{2}\left(\frac{n}{n+1}\right)^{\lambda} \cot \frac{1}{2}\left(\frac{n}{n+1}\right)^{\lambda}\right) \leqslant \\
& \leqslant 1+M_{1}\left(3-\cot 2^{-1}\right) / 2 \approx 1+0,59 M_{1},
\end{aligned}
$$

where $B_{v}$ are the Bernoulli numbers.

$\mathrm{Example} 9$. The Woronoi-Nörlund method $W_{n}$ (special case) is defined by $w_{k}(n):=(n-k+1)(n-k+2) /((n+1)(n+2))$ for $0 \leqslant k \leqslant n$ and $:=0$ for $k>n$. Therefore, it follows that

$$
\begin{aligned}
G\left(w, z^{1} ; n, n\right) & =\frac{2 n+3}{n+2}+M_{1} \frac{n}{n+2} \leqslant 2+M_{1} \\
G\left(z^{1}, w ; n, n\right) & =\frac{n+2}{2 n+3}\left(1+M_{1} \sum_{v=1}^{\infty}\left(\frac{n}{2 n+3}\right)^{v}\right)= \\
& =\frac{n+2}{2 n+3}\left(1+M_{1} \frac{n}{n+3}\right) \leqslant \frac{1}{2}\left(1+M_{1}\right) .
\end{aligned}
$$

Example 10. The Riesz means are defined by $r_{k}^{\lambda, \delta}(n):=(1-$ $\left.-(k /(n+1))^{\lambda}\right)^{\delta} \quad(\lambda>0, \delta>0)$ for $0 \leqslant k \leqslant n$ and $:=0$ for $k>n$. In this example we treat separately three special cases of the Riesz means. 
a) Setting $x=(k /(n+1))^{\lambda}(k \leqslant n)$, the expansion

$$
\frac{1-(1-x)^{\delta}}{x}=\sum_{v=0}^{\infty}(-1)^{v}\left(\begin{array}{c}
\delta \\
v+1
\end{array}\right) x^{v} \quad(\delta>0)
$$

gives

$$
\frac{1-r_{k}^{\lambda, \delta}(n)}{1-z_{k}^{\lambda}(n)}=\sum_{v=0}^{\infty}(-1)^{v}\left(\begin{array}{c}
\delta \\
v+1
\end{array}\right)\left(\frac{k}{n+1}\right)^{\lambda v} \cdots
$$

This result implies

$$
\begin{aligned}
G\left(r^{\lambda, 0}, z^{\lambda} ; n, n\right) & =\delta+M_{1} \sum_{v=1}^{\infty}\left|\left(\begin{array}{c}
\delta \\
v+1
\end{array}\right)\right|\left(\frac{n}{n+1}\right)^{\lambda v} \leqslant \\
& \leqslant \delta+M_{1}\left(2^{\{\delta\}}-\delta-1\right),
\end{aligned}
$$

where $\{\delta\}:=\inf \{k \in \mathbf{P}: k \geqslant \delta\}$.

b) Let $\delta=2,3,4,5$, be fixed. Now we consider the Lagrangian interpolation formula

$$
\frac{x}{1-(1-x)^{\delta}}=-\frac{1}{\delta} \sum_{v=1}^{\delta-1} \frac{b_{v}}{1-x /\left(1-b_{v}\right)},
$$

where $b_{v}:=\cos (2 v \pi / \delta)+i \sin (2 v \pi / \delta)$. From direct calculation it follows that $\left|1-b_{v}\right|=2 \sin (v \pi / \delta)>1$ for all $v \leqslant \delta-1, \delta \leqslant 5$. Due to the geometric series we have

$$
\frac{x}{1-(1-x)^{\delta}}=\frac{1}{\delta}\left(1-\sum_{\mu=1}^{\delta-1} b_{\mu} \sum_{v=1}^{\infty}\left(\frac{x}{1-b_{\mu}}\right)^{v}\right) .
$$

Setting $x=(k /(n+1))^{\lambda}$, we get

$$
\frac{1-z_{k}^{\lambda}(n)}{1-r_{k}^{\lambda, \delta}(n)}=\frac{1}{\delta}\left(1-\sum_{\mu=1}^{\delta-1} b_{\mu} \sum_{v=1}^{\infty}\left(1-b_{\mu}\right)^{-v}\left(\frac{k}{n+1}\right)^{\lambda v}\right)
$$

and thus

$$
\begin{aligned}
G\left(z^{\lambda}, r^{\lambda, \delta} ; n, n\right)= & \frac{1}{\delta}\left(1+M_{1} \sum_{\mu=1}^{\delta-1} \sum_{v=1}^{\infty}\left(2 \sin \frac{\mu \pi}{\delta}\right)^{-v}\left(\frac{n}{n+-1}\right)^{\lambda v}\right) \leqslant \\
\leqslant & \frac{1}{\delta}\left(1+M_{1} \sum_{\mu=1}^{\delta-1}\left(2 \sin \frac{\mu \pi}{\delta}-1\right)^{-1}\right)= \\
& \left(1+M_{1}\right) / 2, \quad \delta=2, \\
= & \left(1+M_{1}(1+\sqrt{3})\right) / 3, \quad \delta=3, \\
& \left(1+M_{1}(3+2 \sqrt{2})\right) / 4, \quad \delta=4, \\
& \left(1+13,6 M_{1}\right) / 5, \quad \delta=5 .
\end{aligned}
$$

c) Let $p=2,3, \ldots, \delta>0$, be fixed. For $|x| \leqslant 1$ we have

$$
\frac{1-(1-x)^{\delta}}{1-(1-x)^{\delta / p}}=p+\sum_{\mu=1}^{p-1} \sum_{v=1}^{\infty}(-1)^{v}\left(\begin{array}{c}
\delta \mu / p \\
v
\end{array}\right) x^{v} .
$$

Setting $x=(k /(n+1))^{\lambda}$, we obtain 


$$
\frac{1-r_{k}^{\lambda, \delta}(n)}{1-r_{k}^{\lambda, \delta / p}(n)}=p+\sum_{\mu=1}^{p-1} \sum_{v=1}^{\infty}(-1)^{v}\left(\begin{array}{c}
\delta \mu / p \\
v
\end{array}\right)\left(\frac{k}{n+1}\right)^{\lambda v}
$$

and

$$
\begin{aligned}
G\left(r^{\lambda, \delta}, r^{\lambda, \delta / p} ; n, n\right) & =p+M_{1} \sum_{\mu=1}^{p-1} \sum_{v=1}^{\infty}\left|\left(\begin{array}{c}
\delta \mu / p \\
v
\end{array}\right)\right|\left(\frac{n}{n+1}\right)^{\lambda v} \leqslant \\
& \leqslant p+M_{1}\left(\sum_{\mu=0}^{p-1} 2^{\{\delta \mu / p\}}-p\right) .
\end{aligned}
$$

With this example we shall conclude our applications. In this way it is possible to treat some other methods of summation (e.g. method of Riemann-Lebesgue etc.), but not in such a complete form as presented above.

\section{REFERENCES}

1. Ахи езер Н. И., Лекции по теории аппроксимации, М., «Наука», 1966.

2. G ör li c h, E., N e s s e l, R. J., T re be ls, W., Acta Sci. math., 34, 121-130 (1973).

3. Butzer, P. L., Nesse1, R. J., Trebe1s, W., Tohoku Math. J., 24, № 2, $127-140(1972)$

4. F a va rd, J., In: On Approximation Theory (P. L. Butzer and J. Korevaar, Eds.), ISNM 5, «Birkhäuser», Basel, 1964, p. 4-11.

5. F a v a r d, J., SIAM J. Number. Anal., Ser B1, 38-52 (1964).

6. Sh a p i ro, H. S., Bull. Amer. Math. Soc., 74, № 3, 500-504 (1968).

7. Т р и г у б Р. М., Изв. АН СССР, Сер. матем., 32, № 1, 24-49 (1968).

8. Z a m a n s ky, M., Bull. sci. math., 101, № 1, 3-70 (1977); 101, № 2, 149-188 (1977).

9. К и вин ук к А., Уч. зап. Тартуск. ун-та, 448, 31-39 (1978).

10. Butze r, P. L., N e s e 1, R. J.; Fourier Analysis and Approximation, I, «Birkhäuser», Basel-Stuttgart, 1971.

Tallinn

Polytechnical Institute
Received

October 26, 1981

\section{A. KIVINUKK}

\section{KONSTANTIDE HINNANGUID SISALDAVAD SUMMEERUVUSMEETODITE VORDLUSTEOREEMID}

Artikkel käsitleb lähendamist Banachi ruumis Fourier' arenduste summeeruvusmeetoditega. On eeldatud, et Banachi ruumis leidub projektorite jada, mille puhul kehtivad Bernšteini (B) ja multiplikaatorite tüüpi $(M)$ võrratused. On tōestatud summeeruvusmeetodite koonduvuskiiruste võrdlusteoreem, mis sisaldab ka vastavate konstantide hinnanguid.

\section{A. КИВИНУКК}

\section{ТЕОРЕМЫ СРАВНЕНИЯ МЕТОДОВ СУММИРОВАНИЯ С ОЦЕНКОИ ПОСТОЯННЫХ}

Рассматриваются приближения в банаховом пространстве методами суммирования разложений Фурье. Предполагается, что в пространстве существует такая последовательность проекторов, при которой имеют место неравенства Бернштейна (В) и неравенства типа мультипликаторов $(M)$. Доказывается теорема сравнения порядка приближения методами суммирования с оценкой постоянных. 